\title{
Acute abdomen in pregnancy, don't forget colonic cancer
}

\author{
Wong Winn Lung, Dharmendra Ganasagaran \\ Corresponding author: Dr. Wong Winn Lung, MD (UKM), Medical officer, Surgery Department, \\ Hospital Melaka, Malaysia; Email: alex.wwl91@gmail.com
}

Distributed under Attribution-Non Commercial - Share Alike 4.0 International (CC BY-NC-SA 4.0)

\begin{abstract}
Colonic cancer is rare during pregnancy. Clinical manifestation of left sided colonic tumour usually resulted in some degree of altered bowel habit. Early diagnosis is difficult as other clinical manifestation of colorectal cancer, such as constipation, abdominal pain or distension are often attributed in normal pregnancy or other gynecological pathology. Here we reported a case of young lady who presented with acute abdomen during pregnancy requiring surgery. She made good recovery post operatively and followed up post chemotherapy.
\end{abstract}

Keywords: Colonic neoplasms, pregnancy, abdominal pain.

Acute abdomen during pregnancy poses great challenge in management of patient, in terms of clinical diagnosis, radiological investigations and treatment. Colorectal cancer in pregnancy is rare, with incidence of 0.8 per 100,000 pregnancies ${ }^{1}$. Diagnosis and treatment of colorectal cancer during pregnancy is often challenging as the signs and symptoms of colon cancer are often attributed to those signs and symptoms of pregnancy. Because of the similar symptoms, there is usually delay in recognition and treatment of colorectal cancer in pregnancy.

\section{Case}

A 29 year old Malay lady (gravida 3 para 2) with antenatal history of anemia in pregnancy (history of requiring blood transfusion), presented at 32 weeks 5 days period of amenorrhea. She presented with 5 day history of left lumbar region pain, physical examination showed abdomen was soft, mild tender left lumbar, vague mass felt at left lumbar region (about $5 \times 5 \mathrm{~cm}$ ). Ultrasonography showed mass at left lumbar $7.5 \mathrm{~cm} \times 6.7 \mathrm{~cm}$, mainly solid component with close proximity to uterus (figure 1). Possible diagnosis of ovarian tumour (possible twisted), pedunculated fibroid, degenerated fibroid were made. During her hospitalisation, she develop severe abdominal pain, obstetrician proceed with exploratory laparotomy with caesarean delivery for indication of suspicion of twisted ovarian tumour. Patient has no complaint of altered bowel habit. The family history was negative for gastrointestinal disease and colorectal cancer.

Initial investigations revealed leucocytosis (WBC $\left.17.8^{\wedge} 9 / \mathrm{L}\right)$ with anemia $(\mathrm{Hb} 9.8 \mathrm{~g} / \mathrm{L})$ and other biochemical bloods were within normal range. Urine dipstix and microscopic examination was normal.

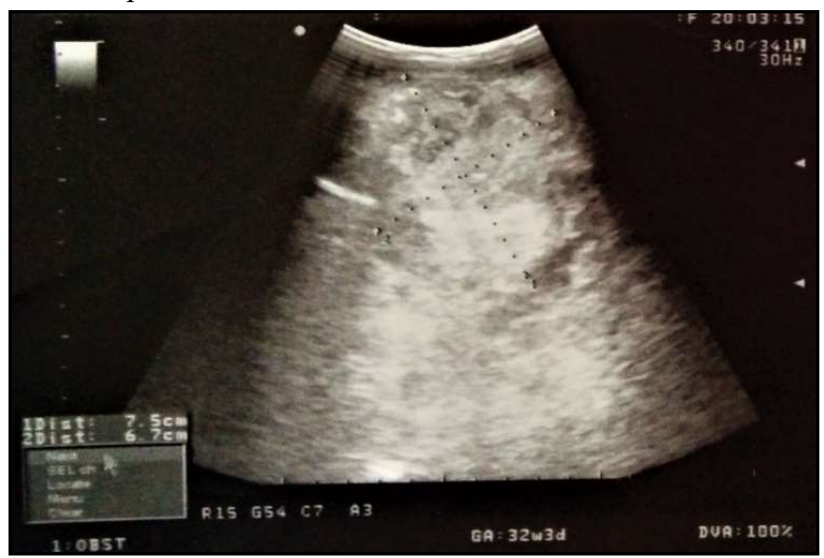

Figure 1: Ultrasonography of left lumbar mass, measuring 7.5 x $6.7 \mathrm{~cm}$.

On exploratory laparotomy examination, it was revealed that uterus was normal. Caesarean delivery was performed. Right ovary and fallopian tube was normal. Left ovary was

Received: $3^{\text {rd }}$ February 2021, Peer review completed: $14^{\text {th }}$ May 2021, Accepted: $7^{\text {th }}$ June 2021.

Lung WW, Ganasagaran D. Acute abdomen in pregnancy, don't forget colonic cancer. The New Indian Journal of OBGYN. 2022; 8(2): 311 - 14. 
inflammed and adhered to bowel mass over left side. The surgery team was called in, they noted sealed perforated descending colon tumour, adhered to lateral abdominal wall with multiple enlarged mesenteric nodes. Decision to proceed with left hemicolectomy was taken and executed in the usual manner via open surgery (figure 2). Primary bowel anastomosis was performed post colectomy. The patient developed superficial surgical site infection which resolved and made good recovery, was discharge on day 10 post operatively. On further inspection, gross specimen showed constricting tumour with perforation (figure 3 ).

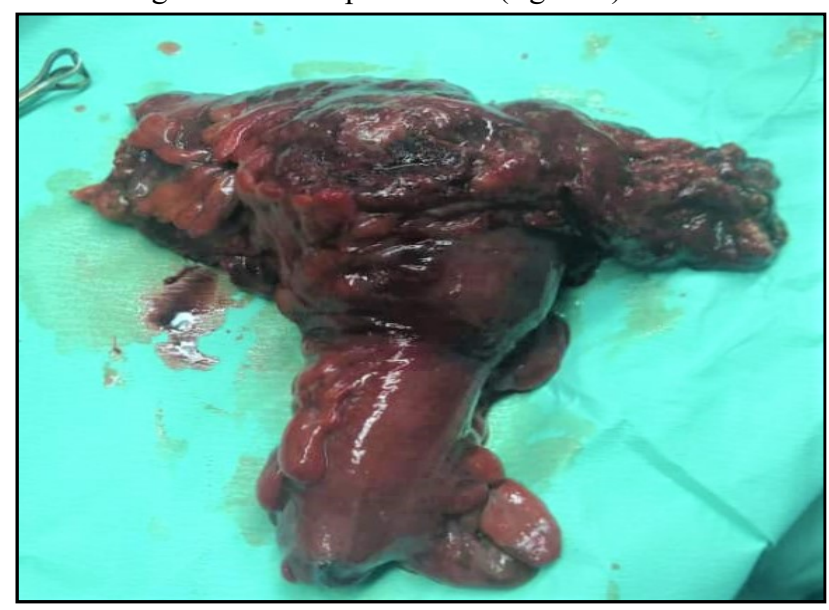

Figure 2: Resected descending colon (left hemicolectomy).

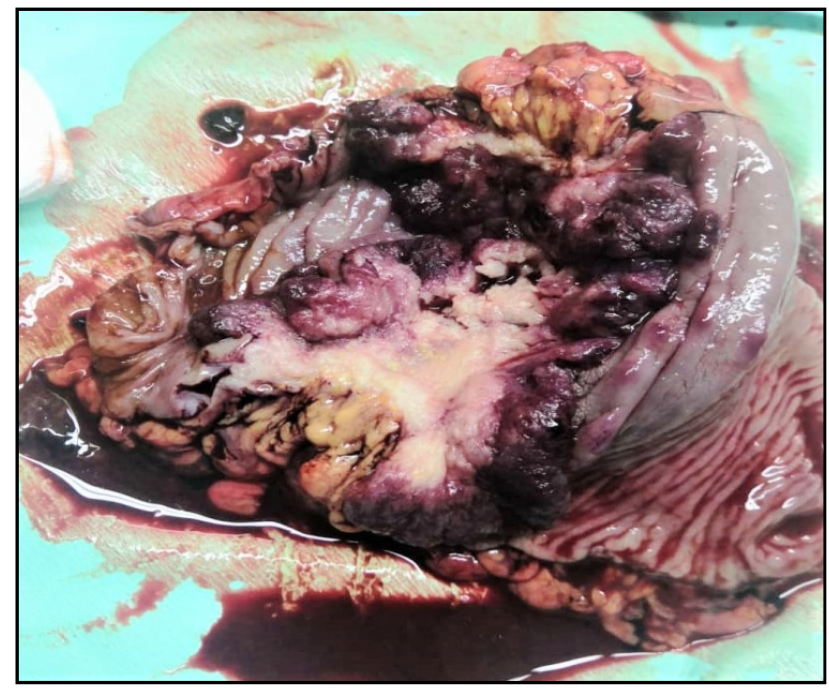

Figure 3: The colon is cut open revealed constricting tumour with multiple perforation site.

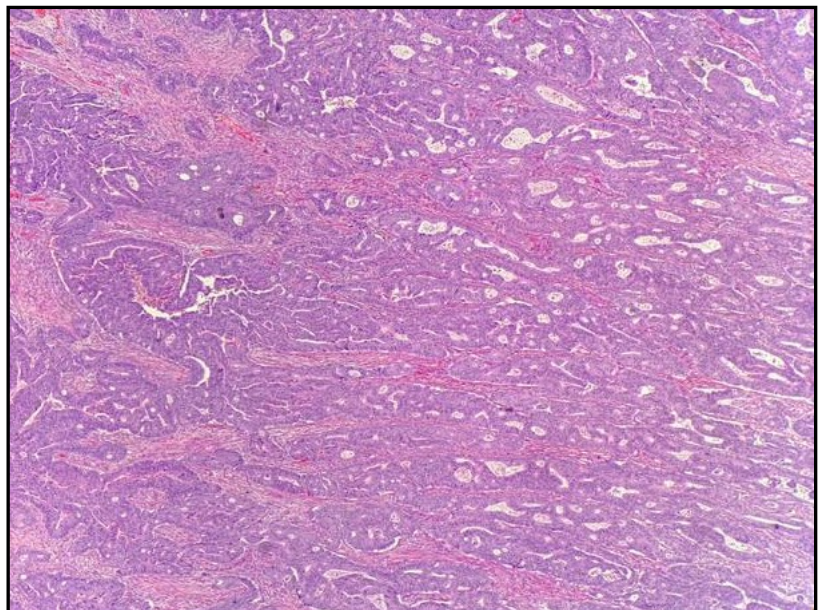

Figure 4: Histological appearance of adenocarcinoma of descending colon (Hematoxylin and Eosin stain under 40x magnifications).

Subsequently, patient was managed together with oncologist. Histology finding was moderate differentiated adenocarcinoma (figure 4, 5), tumour perforation identified with abscess in surrounding area. There was complete resection of tumour at all margin, there was local invasion beyond muscularis propria, 8 of 15 lymph nodes were involved. Modified Dukes stage was given C1 (pT4, pN2b, $\mathrm{pMx}$ ). Complete staging was performed post operatively. TNM staging pT4N2bM0, staging IIIc. Patient then received total 8 cycles of neoadjuvant chemotherapy (IV Oxaliplatin and $\mathrm{T}$ Capecitabine).

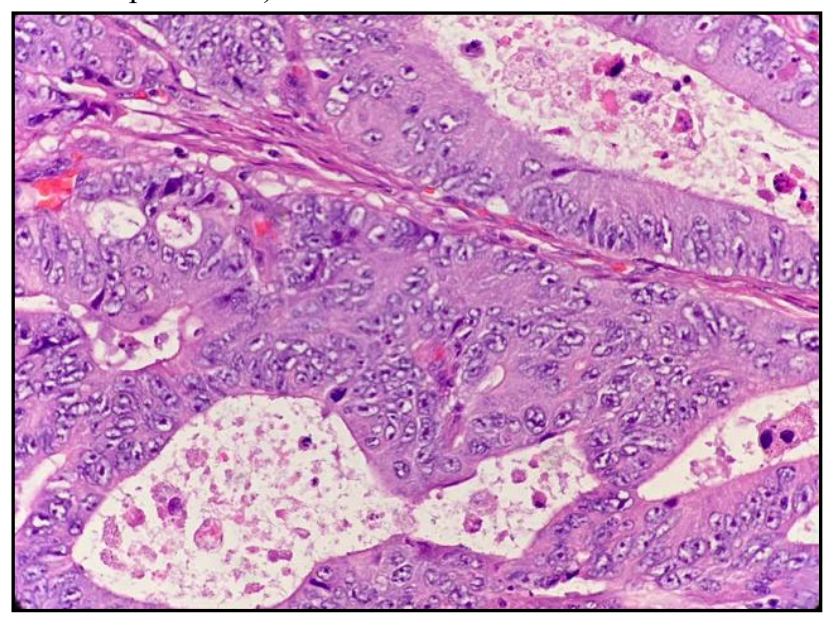

Figure 5: Histological appearance of adenocarcinoma of descending colon (Hematoxylin and Eosin stain under 400x magnifications). 


\section{Discussion}

In general population, colorectal cancer is the third most commonly diagnosed cancer ${ }^{2}$. In women, colorectal cancer is the third leading cause of cancer death $(9.5 \%$ of all cancer death $)^{2}$. Colorectal cancer in pregnancy still remain rare, with incidence of 0.8 per 100,000 pregnancies $^{1}$. In a report showed that mean age of female having colorectal cancer during pregnancy was 31 years of age with age range of sixteen to forty-eight ${ }^{3}$.

In a study carried out by P Kocian et al in 2008, most patients present non-acutely. In ten patients out of forty-one patients studied, the diagnosis of colorectal cancer was made during acute surgery. They underwent clinical diagnosis of acute bowel obstruction, bowel perforation and suspicion of ovarian pathology like torsion. Rest of the patients presented with complaints of rectal bleeding, abdominal pain, change in bowel habits, weight loss, abdominal mass ${ }^{4}$. Patient in our case study presented solely with complaint of abdominal pain, no prior altered bowel habits or constitutional symptoms, these made clinical diagnosis of colorectal tumour less favourable as compared to other gynecological pathology.

There is often delay in diagnosis as initial symptoms of colorectal cancer are presumed to be attributed to normal pregnancy. Obtaining complete history and thorough physical examination, including digital rectal examination still remained as key component in early diagnosis of colorectal cancer in pregnancy ${ }^{5}$. Endoscopy examination raises the issue of fetal safety which include risk of maternal oversedation, fetal exposure to potential teratogenic medication, uteroplacental insufficiency due to maternal positioning that may lead to inferior vena cava compression by uterus ${ }^{6-8}$. Therefore, endoscopy examination during pregnancy should only be performed with strong indication and to be delayed until second trimester of pregnancy whenever possible ${ }^{8}$. Further assessment of pregnant patients diagnosed with colorectal carcinoma is relatively similar to those general population ${ }^{9}$. In this case study, the diagnosis of descending colon tumour was made intraoperatively during exploration.

Treatment in colorectal carcinoma during pregnancy is largely influenced by gestational duration. Radical surgery of the tumour should be the main aim in patient presenting during first 6 months of pregnancy ${ }^{10}$. According to some studies, surgery can be delayed until delivery of the infant with maternal risk of tumour progression, if diagnosis of colorectal cancer is made after 20 weeks gestation ${ }^{9}$. In patient presenting with bowel obstruction, tumour resection with or without primary anastomosis is recommended if patient presented in early gestation, while those presented late, colostomy followed by tumour resection post delivery is recommended ${ }^{7}$.

Delivery mode by either cesarean section or per vaginally depends on obstetric indication and presence of delivery canal obstruction, colorectal cancer itself is not an indication to deliver via cesarean section ${ }^{11}$. Resection of colorectal tumour can be performed during period of delivery if cesarean section is the chosen method of delivery ${ }^{11}$. In this case study, cesarean section was planned prior laparotomy exploration, thus colorectal tumour resection was performed in the same setting.

\section{Conclusion}

Early recognition and diagnosing colorectal cancer in pregnancy is important, albeit rare. Clinician should raise suspicion of possible colorectal cancer when pregnant patient presented with acute abdomen. Treatment and therapy should be individualized and would be best managed by multidisciplinary team.

\section{Conflict of interest: None. Disclaimer: Nil.}

\section{References}

1. Lee YY, Roberts CL, Dobbins T, et al. Incidence and outcomes of pregnancy-associated cancer in Australia, 1994-2008: a population-based linkage study. BJOG. 2012; 119:1572-82

2. Latest global cancer data: Cancer burden rises to 18.1 million new cases and 9.6 million cancer deaths in 2018 . World Health Organization (WHO). 12 Sept 2018.

3. Bernstein MA, Madoff RD, Caushaj PF. Colon and rectal cancer in pregnancy. Dis Colon Rectum. 1993 Feb; 36(2): 172-8

4. Kocian P, de Haan J, Cardonick EH, et al. Management and outcome of colorectal cancer during pregnancy: report of 41 cases. Acta Chirurgica Belgica. 2019; 119: $166-75$.

5. Heise RH, Van Winter JT, Wilson TO, Ogburn Jr PL. Colonic Cancer During Pregnancy: A Case Report and Review of the Literature. Mayo Clin Proc. 1992; 67:1180-84.

6. Cappell MS. Gastrointestinal endoscopy in high-risk patients. Dig Dis. 1996 Jul-Aug; 14(4): 228-44.

7. Longo SA, Moore RC, Canzoneri RJ, Robichaux A. Gastrointestinal Conditions during Pregnancy. Clinics in Colon and Rectal Surgery. 2010 Jun; 23(2): 80-9. 
The New Indian Journal of OBGYN. 2021 (January-June);8(2)

8. Yaghoobi M, Koren G, Nulman I. Challenges to diagnosing colorectal cancer during pregnancy. Can Fam Physician. 2009; 55(9): 881-85.

9. Walsh C, Fazio VW. Cancer of the colon, rectum and anus during pregnancy. The surgeon's perspective. Gastroenterol Clin North Am. 1998 Mar; 27(1): 257-67.

10. Heres $\mathrm{P}$, Wiltink J, Cuesta MA, Burger $\mathrm{CW}$, van Groeningen CJ, Meijer S. Colon carcinoma during pregnancy: A lethal coincidence. European Journal of Obstetrics \& Gynecology and Reproductive Biology. 1993. 48(2): 149-52.
11. Makoshi Z, Perrott C, Al-Khatani K, Al-Mohaisen F. Chemotherapeutic treatment of colorectal cancer in pregnancy: case report. BMC Journal of Medical Case Reports. 2015 June 13; 9: 140.

Wong Winn Lung ${ }^{1}$, Dharmendra Ganasagaran ${ }^{2}$

${ }^{1}$ Medical officer, Surgery Department, Hospital Melaka, Malaysia; ${ }^{2}$ MBBS, MS (Surgery), Surgery Department, Hospital Melaka, Malaysia. 